\title{
REVIEW
}

\section{Review on Malaysian Goniothalamus essential oils and their comparative study using multivariate statistical analysis}

\author{
Natasa Mohd Shakri ${ }^{1}$, (iD) Wan Mohd Nuzul Hakimi Wan Salleh ${ }^{1, *}$ and (iD) Shazlyn Milleana \\ Shaharudin ${ }^{2}$ \\ ${ }^{1}$ Department of Chemistry, Faculty of Science and Mathematics, Universiti Pendidikan Sultan Idris (UPSI), 35900 Tanjong \\ Malim, Perak, MALAYSIA \\ ${ }^{2}$ Department of Mathematics, Faculty of Science and Mathematics, Universiti Pendidikan Sultan Idris (UPSI), 35900 \\ Tanjong Malim, Perak, MALAYSIA
}

*Corresponding author. Email: wmnhakimi@fsmt.upsi.edu.my

Submitted: 27.08.2020; Accepted: 24.12.2020

\begin{abstract}
The genus Goniothalamus is belonging to the Annonaceae family, consists of ca. 2500 species and found in tropical Southeast Asia. The Goniothalamus essential oils were recognized to possess considerable biological activities with varied chemical composition. This article aims to overview the medicinal uses, chemical compositions, and biological activities of Malaysian Goniothalamus essential oils considered as a medicinal plants, widely used as traditional herbal medicines in the treatment of various diseases. The data were collected from the scientific electronic databases including SciFinder, Scopus, Elsevier, PubMed and Google Scholar. Ten Goniothalamus species have been reported for their essential oils and biological activities. It can be observed that the major components were $\alpha$-cadinol, terpinen-4-ol, $\beta$-eudesmol, $\beta$-selinene, linalool, limonene, $\alpha$-copaene, 1,8 -cineole and $\beta$-cubebene. In addition, the selected chemical components from the bark, leaf and root oils were analysed using Principal Component Analysis (PCA) and Hierarchical Cluster Analysis (HCA) and were able to cluster in four groups based on relationships and chemical patterns in essential oils. This multivariate data analysis may be used for the identification and characterization of essential oils from different Goniothalamus species that are to be used as raw materials of traditional herbal products.
\end{abstract}

Keywords: Annonaceae, Goniothalamus, essential oil, principal component analysis, hierarchical cluster analysis

\section{Introduction}

Known as the most powerful therapeutic agents, essential oils are usually used as an alternative medicine known as aromatherapy to support human health and well-being. Additionally, essential oils have also been commonly used in the cosmetics, food, and agricultural industries. Essential oils are usually extracted from a natural source and have that particular plant's fragrance (Winska et al., 2019; Salleh et al., 2014a). The essential oil is normally stored within the plant's oil cells, glands, and vessels. It is released from the flowers as a fragrant fragrance, or retained in the plant's seeds, fruits, leaves, barks or roots until it gradually evaporates (Burger et al., 2019; Salleh et al., 2014b). Essential oils from aromatic and medicinal plants have been known since antiquity to possess biological activity, most notably antibacterial, antifungal and antioxidant properties (Salleh et al., 2015a, 2015b, 2015c, 2016a, 2016b, 2016c). Annonaceae is one of the plant families which numerous report on essential oils, due to its wide use in various traditional medicines and believed to have high medicinal values.

Annonaceae family is the largest family of the order Magnoliales consisting of approximately 135 genera and more than 2,500 species. The family has a source of edible fruit that can be considered to have economic importance. Goniothalamus is a genus of the Annonaceae family, with approximately 160 species of trees and shrubs frequently found in tropical Southeast Asia throughout Indochina and Malaysia (Anary et al., 2016; Aslam et al., 2016). The botanical characteristics are simple, strongly aromatic bark, having few leaves that are simple, alternate and exstipulate. The secondary nerves are also oblique, straight and parallel to 
scalariform reticulations. The axillary flowers are characteristically woody, fusiform and often dark green (Wiart, 2006, 2007; Nielsen, 1993). Goniothalamus species have been used as traditional medicines in Malaysia but information about the volatile composition quality of essential oils from these herbal materials is still limited. Previous studies have described the biological activities of various Goniothalamus species such as antibacterial (Funnimid et al., 2019), antimicrobial (Ghani et al., 2010), antifungal (Duc et al., 2016), antioxidant (Iqbal et al., 2015), and cytotoxicity activities (Kim et al., 2013). The available information on the essential oils of Goniothalamus species was collected via electronic searches such as Pubmed, SciFinder, Scopus, Google Scholar, and Web of Science.

This work aims to give an overview of all published studies on the chemical composition and biological activities of Malaysian Goniothalamus essential oils. In addition, the multivariate statistical analysis was also determined for the leaf, bark and root oils of selected Goniothalamus species. Principal component analysis (PCA) and hierarchical cluster analysis (HCA) were used to characterize their essential oils components.

\section{Traditional uses}

Goniothalamus species are used to induce abortion, antiaging, body pain, rheumatism, skin irritation, typhoid fever, tympanites, stomach ache and fever in widespread medicinal commodities. Table 1 shows several Goniothalamus species and their medicinal uses.

Table 1. Medicinal uses of several Goniothalamus species

\begin{tabular}{|c|c|c|c|}
\hline Species & Local name & Part & Medicinal uses \\
\hline \multirow[t]{2}{*}{ G. amuyon } & amúyon & Seeds & Used to treat scabies, rheumatism and tympanites (Ahmad et al., 1991) \\
\hline & & Fruits & Used to treat stomachache (Quisumbing, 1951) \\
\hline G. cheliensis & & Stems & $\begin{array}{l}\text { Used for the treatment of liver cancer, lung carcinoma and chronic cough } \\
\text { (Jiang et al., 2011) }\end{array}$ \\
\hline G. dolichocharpus & bihidieng & Roots & $\begin{array}{l}\text { It is boiled and taken orally by the Kelabit community to ease stomachache } \\
\text { (Quisumbing, 1951) }\end{array}$ \\
\hline \multirow[t]{2}{*}{ G. giganteus } & penawar hitam & Roots & Used in abortion and treatment of colds (Wiart, 2006) \\
\hline & & Leaves & Heated leaves are applied to swellings (Wiart, 2006) \\
\hline G. lanceolatus & selukai & Leaves & $\begin{array}{l}\text { Used as a traditional remedy for fever, skin infection, postpartum, abortion, } \\
\text { as well as a cancer treatment (Wiart, 2007) }\end{array}$ \\
\hline G. laoticus & Khao Lam-dong & Stem bark & $\begin{array}{l}\text { Used traditionally as a tonic and a febrifuge by the local people in the } \\
\text { northeastern part of Thailand (Wu et al., 1991) }\end{array}$ \\
\hline \multirow[t]{2}{*}{ G. macrophyllus } & selayak hitam & Leaves & Used to allay fever (Alkofahi et al., 1988) \\
\hline & & Roots & $\begin{array}{l}\text { Used as a postpartum remedy and to cause abortion, antiaging purposes, } \\
\text { rheumatisms, skin complaints, eliminate excessive gas in the body and used } \\
\text { as a lotion to treat body pains (Alkofahi et al., 1988) }\end{array}$ \\
\hline G. marcanii & & Leaves & $\begin{array}{l}\text { Used treating for infectious diseases in early childhood (Mahiwan et al., } \\
\text { 2013) }\end{array}$ \\
\hline \multirow[t]{2}{*}{ G. malayanus } & kenanga paya & Roots & Used for the treatment of rheumatism and fever (Ahmad et al., 1991) \\
\hline & & Stem bark & Used to treat measles and as insect repellents (Ahmad et al., 1991) \\
\hline G. scortechinii & gajah beranak & Leaves & $\begin{array}{l}\text { Used as a postpartum protective remedy and were used to improve blood } \\
\text { circulation (Burkill, 1966) }\end{array}$ \\
\hline G. sesquipedalis & & Leaves & $\begin{array}{l}\text { Used to treat fever, cough, colds, snakebite, pains, and infectious and } \\
\text { inflammatory diseases (Akter et al., 2018) }\end{array}$ \\
\hline
\end{tabular}




\begin{tabular}{|c|c|c|c|}
\hline G. tapis & kenarak & Roots & $\begin{array}{l}\text { Used as an abortifacient during early months of pregnancy, an infusion of } \\
\text { the roots is used to treat typhoid fever (Inayat-Hussain et al., 1999) }\end{array}$ \\
\hline G. uvarioides & belindung & Roots & $\begin{array}{l}\text { Used as postpartum protective remedies, abortifacients, and to treat } \\
\text { typhoid fever, rheumatism, and headache (Moharam et al., 2012) }\end{array}$ \\
\hline G. velutinus & kayu tas hitam & Leaves & $\begin{array}{l}\text { Used as a traditional medicine for treating headache, food poisoning, as } \\
\text { well as snakebite remedies, induce abortion and as a post-partum remedy } \\
\text { (Inayat-Hussain et al., 1999) }\end{array}$ \\
\hline
\end{tabular}

\section{Chemical composition of Malaysian Goniothalamus essential oils}

There are ten species of Goniothalamus originated from Malaysia that were successfully reported for their essential oil composition. The essential oils of ten local Goniothalamus which were $G$. andersonii, $G$. clemensii, G. macrophyllus, G. malayanus, G. ridleyi, G. tapis, G.tapisoides, G. velutinus, G. uvarioides, and $G$. woodii have been investigated (Jusoh et al., 2015; Moharam et al., 2010; Ghani et al., 2010; Ahmad et al., 2010; Jantan et al., 2005). The plant parts used for the extraction of essential oils includes flower, fruit, leaf, stem, bark, and root. However, most of the essential oils from Malaysian Goniothalamus were extracted from bark, leaf and root. Table 2 shows the major components identified in Goniothalamus essential oils, whereas Figure 1 so its chemical structures.

Table 2. Major components identified in Malaysian Goniothalamus essential oils

\begin{tabular}{|c|c|c|c|c|c|c|}
\hline Species & Locality & Parts & $\begin{array}{l}\text { Yield } \\
(\%)\end{array}$ & $\begin{array}{l}\text { Total } \\
\text { components } \\
(\%)\end{array}$ & Major components & References \\
\hline G. andersonii & Sarawak & Leaf & 0.70 & $25(85.30)$ & $\begin{array}{l}\text { Guaiol (28.60\%) (1), elemol } \\
(19.60 \%), \beta \text {-caryophyllene }(7.70 \%)\end{array}$ & $\begin{array}{c}\text { Jantan et al., } \\
2005\end{array}$ \\
\hline G. clemensii & Sarawak & Bark & 2.80 & $23(98.10 \%)$ & $\begin{array}{l}\text { a-Cadinol (41.60\%) (2), agarospirol } \\
(19.00 \%) \text {, elemol (16.10\%) }\end{array}$ & $\begin{array}{l}\text { Moharam et } \\
\text { al., } 2010\end{array}$ \\
\hline G. macrophyllus & N. Sembilan & Root & 0.05 & $14(42.50 \%)$ & $\begin{array}{l}\text { Cyperene (9.80\%) (3), geranyl } \\
\text { acetate }(9.40 \%) \text {, camphene }(7.50 \%)\end{array}$ & $\begin{array}{c}\text { Ghani et al., } \\
2010\end{array}$ \\
\hline G. macrophyllus & N. Sembilan & Twig & 0.14 & $21(90.00 \%)$ & $\begin{array}{l}\text { Geranyl acetate }(45.50 \%) \quad(4), \\
\text { geraniol }(17.00 \%), \quad \text { linalool } \\
(12.70 \%) \text {, camphene }(7.50 \%)\end{array}$ & $\begin{array}{c}\text { Ghani et al., } \\
2010\end{array}$ \\
\hline G. macrophyllus 1 & Pahang & Bark & 0.80 & $42(97.80 \%)$ & $\begin{array}{l}\text { Terpinen-4-ol (38.80\%) (5), 1,8- } \\
\text { cineole }(18.10 \%) \text {, geranyl acetate } \\
(11.10 \%) \text {, geraniol }(9.70 \%)\end{array}$ & $\begin{array}{c}\text { Jantan et al., } \\
2005\end{array}$ \\
\hline G. macrophyllus 2 & Sarawak & Bark & 0.70 & $41(96.40 \%)$ & $\begin{array}{l}\text { Terpinen-4-ol (42.70\%) (5), } \beta \text { - } \\
\text { ocimene }(25.40 \%), \quad \alpha \text {-terpineol } \\
(10.00 \%), 1,8 \text {-cineole }(5.80 \%)\end{array}$ & $\begin{array}{c}\text { Jantan et al., } \\
2005\end{array}$ \\
\hline G. malayanus & Sarawak & Bark & 0.96 & 36 (93.90\%) & 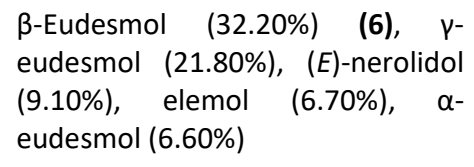 & $\begin{array}{c}\text { Jantan et al., } \\
2005\end{array}$ \\
\hline G. malayanus 1 & Sarawak & Leaf & 0.32 & $35(86.30 \%)$ & $\begin{array}{l}\beta \text {-Selinene (33.60\%) (7), viridiflorol } \\
(13.10 \%) \text {, epiglobulol (7.70\%) }\end{array}$ & $\begin{array}{c}\text { Jantan et al., } \\
2005\end{array}$ \\
\hline G. malayanus 1 & Sarawak & Root & 0.18 & $36(90.50 \%)$ & 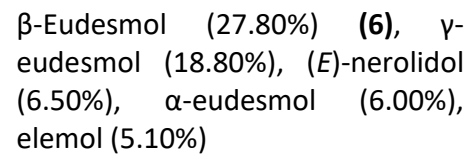 & $\begin{array}{c}\text { Jantan et al., } \\
2005\end{array}$ \\
\hline G. malayanus 2 & Sarawak & Leaf & 0.32 & 35 (86.20\%) & $\begin{array}{l}\beta \text {-Selinene (33.60\%) (7), viridiflorol } \\
(13.10 \%) \text {, epiglobulol (7.70\%) }\end{array}$ & $\begin{array}{c}\text { Jantan et al., } \\
2005\end{array}$ \\
\hline
\end{tabular}




\begin{tabular}{|c|c|c|c|c|c|c|}
\hline G. malayanus 2 & Sarawak & Root & 0.18 & 35 (90.00\%) & 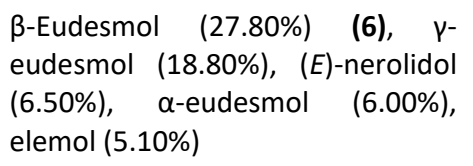 & $\begin{array}{c}\text { Jantan et al., } \\
2005\end{array}$ \\
\hline $\begin{array}{l}\text { G. ridleyii } 1 \\
\text { (Fresh) }\end{array}$ & Kelantan & Bark & 0.11 & $50(89.50 \%)$ & $\begin{array}{l}\text { Linalool }(15.20 \%) \quad(8) \text {, citronellal } \\
(10.90 \%), \quad \beta \text {-eudesmol }(9.80 \%) \text {, } \\
\text { limonene }(7.50 \%) \text {, elemol }(7.40 \%)\end{array}$ & $\begin{array}{l}\text { Jusoh et al., } \\
2015\end{array}$ \\
\hline $\begin{array}{l}\text { G. ridleyii } 1 \\
\text { (Fresh) }\end{array}$ & Kelantan & Stem & 0.03 & 47 (90.10\%) & 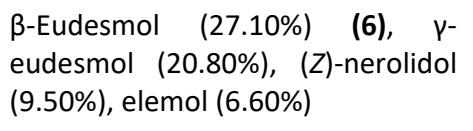 & $\begin{array}{l}\text { Jusoh et al., } \\
2015\end{array}$ \\
\hline $\begin{array}{l}\text { G. ridleyii } 1 \\
\text { (Fresh) }\end{array}$ & Kelantan & Fruit & 0.12 & 49 (89.80\%) & 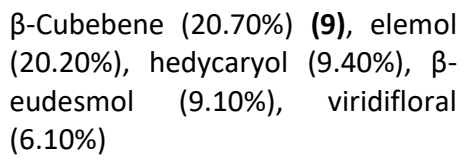 & $\begin{array}{l}\text { Jusoh et al., } \\
2015\end{array}$ \\
\hline G. ridleyii 2 (Dry) & Kelantan & Bark & 0.38 & $39(94.60 \%)$ & $\begin{array}{l}\text { Linalool (15.80\%) (8), citronellal } \\
(12.90 \%) \text {, limonene }(10.60 \%), \quad \beta \text { - } \\
\text { eudesmol }(7.60 \%) \text {, safrole }(7.50 \%)\end{array}$ & $\begin{array}{l}\text { Jusoh et al., } \\
2015\end{array}$ \\
\hline G. ridleyii 2 (Dry) & Kelantan & Leaf & 0.90 & 48 (95.10\%) & $\begin{array}{l}\text { Linalool }(23.40 \%) \quad(8), \quad \alpha \text {-copaene } \\
(19.80 \%), \quad \beta \text {-caryophyllene } \\
(11.40 \%), \quad 1,8 \text {-cineole }(7.00 \%), \\
\text { terpinen-4-ol }(6.10 \%)\end{array}$ & $\begin{array}{l}\text { Jusoh et al., } \\
2015\end{array}$ \\
\hline G. ridleyii 2 (Dry) & Kelantan & Stem & 0.03 & 35 (93.30\%) & 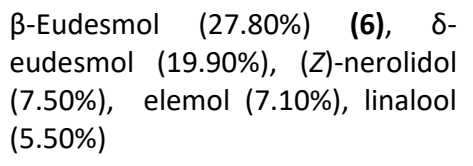 & $\begin{array}{l}\text { Jusoh et al., } \\
2015\end{array}$ \\
\hline G. ridleyii 2 (Dry) & Kelantan & Branch & 0.27 & 37 (93.10\%) & $\begin{array}{l}\beta \text {-Selinene (35.90\%) (7), viridifloral } \\
(16.30 \%),(Z) \text {-nerolidol }(5.90 \%)\end{array}$ & $\begin{array}{l}\text { Jusoh et al., } \\
2015\end{array}$ \\
\hline G. ridleyii 2 (Dry) & Kelantan & Root & 0.15 & 47 (92.80\%) & 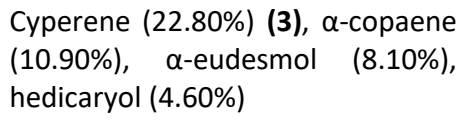 & $\begin{array}{l}\text { Jusoh et al., } \\
2015\end{array}$ \\
\hline G. ridleyii 2 (Dry) & Kelantan & Fruit & 0.83 & 37 (93.20\%) & $\begin{array}{l}\beta \text {-Cubebene }(17.20 \%)(9), \text { elemol } \\
\begin{array}{l}(15.90 \%), \quad \beta \text {-eudesmol }(9.50 \%), \\
\text { hedicaryol } \quad(8.50 \%), \quad \text { viridifloral } \\
(8.40 \%)\end{array}\end{array}$ & $\begin{array}{l}\text { Jusoh et al., } \\
2015\end{array}$ \\
\hline G. tapis & Sarawak & Root & 0.98 & $36(88.00 \%)$ & 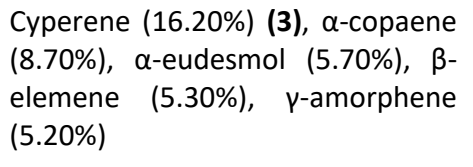 & $\begin{array}{c}\text { Ahmad et al., } \\
2010\end{array}$ \\
\hline G. tapis 1 & Sarawak & Bark & 2.85 & $23(82.70 \%)$ & 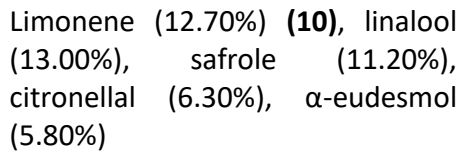 & $\begin{array}{l}\text { Moharam et } \\
\text { al., } 2010\end{array}$ \\
\hline G. tapis 1 & Sarawak & Leaf & 2.23 & 26 (92.40\%) & $\begin{array}{l}\alpha \text {-Copaene }(23.80 \%) \text { (11), linalool } \\
(18.50 \%), \quad \beta \text {-caryophyllene } \\
(14.20 \%), 1,8 \text {-cineole }(7.60 \%)\end{array}$ & $\begin{array}{l}\text { Moharam et } \\
\text { al., } 2010\end{array}$ \\
\hline G. tapis 2 & Sarawak & Bark & 2.85 & $22(83.00 \%)$ & 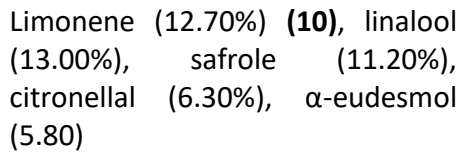 & $\begin{array}{c}\text { Ahmad et al., } \\
2010\end{array}$ \\
\hline G. tapis 2 & Sarawak & Leaf & 2.23 & 29 (93.60\%) & $\begin{array}{l}\alpha \text {-Copaene }(23.80 \%) \text { (11), linalool } \\
(18.50 \%), \quad \beta \text {-caryophyllene } \\
(14.40 \%), 1,8 \text {-cineole }(7.60 \%)\end{array}$ & $\begin{array}{c}\text { Ahmad et al., } \\
2010\end{array}$ \\
\hline G. tapisoides 1 & Sarawak & Bark & 3.86 & $23(99.00 \%)$ & $\begin{array}{lcr}\text { 1,8-Cineole } & (47.90 \%) & (12), \\
\text { terpinen-4-ol } & (22.50 \%), \quad p- \\
\text { menthene }(6.90 \%), \quad \alpha \text {-pinene } \\
(6.60 \%), \nu \text {-terpinene }(6.60 \%)\end{array}$ & $\begin{array}{l}\text { Moharam et } \\
\text { al., } 2010\end{array}$ \\
\hline
\end{tabular}




\begin{tabular}{|c|c|c|c|c|c|c|}
\hline G. tapisoides 1 & Sarawak & Leaf & 3.05 & 26 (99.40\%) & $\begin{array}{l}\text { 1,8-Cineole (79.00\%) (12), } \alpha \text {-pinene } \\
(9.60 \%), \alpha \text {-terpineol (4.40\%) }\end{array}$ & $\begin{array}{l}\text { Moharam et } \\
\text { al., } 2010\end{array}$ \\
\hline G. tapisoides 1 & Sarawak & Root & 1.45 & 20 (99.70\%) & $\begin{array}{l}\text { 1,8-Cineole } \quad(56.10 \%) \quad(12), \\
\text { terpinen-4-ol }(19.60 \%), \gamma \text {-terpinene } \\
(5.70 \%)\end{array}$ & $\begin{array}{c}\text { Moharam et } \\
\text { al., } 2010\end{array}$ \\
\hline G. tapisoides 2 & Sarawak & Bark & 3.86 & $24(92.60 \%)$ & $\begin{array}{l}\text { 1,8-Cineole }(47.90 \%) \quad(12), \\
\text { terpinen-4-ol }(22.50 \%), \alpha \text {-pinene } \\
(6.60 \%), v \text {-terpinene }(6.60 \%)\end{array}$ & $\begin{array}{c}\text { Ahmad et al., } \\
2010\end{array}$ \\
\hline G. tapisoides 2 & Sarawak & Leaf & 3.05 & $28(99.50 \%)$ & $\begin{array}{l}\text { 1,8-Cineole (79.00\%) (12), } \alpha \text {-pinene } \\
(9.60 \%), \alpha \text {-terpineol (4.40\%) }\end{array}$ & $\begin{array}{c}\text { Ahmad et al., } \\
2010\end{array}$ \\
\hline G. tapisoides 2 & Sarawak & Root & 1.45 & 19 (95.60\%) & $\begin{array}{l}\text { 1,8-Cineole } \quad(56.10 \%) \quad(12), \\
\text { terpinen-4-ol }(19.6 \%), y \text {-terpinene } \\
(5.70 \%)\end{array}$ & $\begin{array}{c}\text { Ahmad et al., } \\
2010\end{array}$ \\
\hline G. uvarioides & Sarawak & Leaf & 0.27 & 51 (92.10\%) & $\begin{array}{l}\beta \text {-Cubebene }(15.20 \%)(9) \text {, elemol } \\
(9.70 \%) \text {, epi- } \alpha \text {-cadinol }(6.20 \%) \alpha \text { - } \\
\text { muurolene }(4.80 \%)\end{array}$ & $\begin{array}{c}\text { Jantan et al., } \\
2005\end{array}$ \\
\hline G. uvarioides & Sarawak & Bark & 0.98 & 28 (87.20\%) & 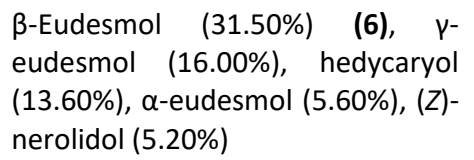 & $\begin{array}{c}\text { Jantan et al., } \\
2005\end{array}$ \\
\hline G. uvarioides & Sarawak & Root & 0.35 & $28(85.50 \%)$ & $\begin{array}{l}\text { Terpinen-4-ol (39.50\%) (5), } 1,8 \text { - } \\
\text { cineole } \quad(14.00 \%), \quad \alpha \text {-terpineol } \\
(6.30 \%), p \text {-cymene }(5.10 \%)\end{array}$ & $\begin{array}{c}\text { Jantan et al., } \\
2005\end{array}$ \\
\hline G. velutinus & Sarawak & Bark & 1.40 & 45 (93.90\%) & $\begin{array}{l}\alpha \text {-Cadinol (14.00\%) (2), } \alpha \text {-eudesmol } \\
\begin{array}{l}(9.70 \%), \quad \text { t-muurolol } \quad(9.10 \%), \quad \beta- \\
\text { selinene } \\
(5.20 \%)\end{array} \quad\end{array}$ & $\begin{array}{l}\text { Moharam et } \\
\text { al., } 2010\end{array}$ \\
\hline G. woodii & Sarawak & Bark & 1.80 & 36 (97.00\%) & $\begin{array}{l}\alpha \text {-Cadinol (21.90\%) (2), elemol } \\
(12.60 \%) \text {, agarospirol }(8.00 \%)\end{array}$ & $\begin{array}{l}\text { Moharam et } \\
\text { al., } 2010\end{array}$ \\
\hline
\end{tabular}

Figure 1. Chemical structures of several major components identified from Goniothalamus essential oils.<smiles>C[C@H]1CCC2=C(C[C@H](C(C)(C)C)CC2)[C@@H]1C</smiles>

(1)<smiles>CC1=CCC(O)(C(C)C)CC1</smiles>

(5)<smiles></smiles>

(9)<smiles>CC1=C[C@H]2[C@@H](C(C)C)CC[C@H](C)[C@H]2CC1</smiles>

(2)<smiles>C=C1CCC[C@]2(C)CC[C@@H](C(C)(C)O)C[C@@H]12</smiles>

(6)<smiles>C=C(C)[C]1CC=C(C)CC1</smiles>

(10)<smiles>CC1=C2CC3CC[C@H](C)C2(CC1)C3(C)C</smiles>

(3)<smiles>C=C1CCC[C@]2(C)CC[C@@H](C(=C)C)C[C@H]12</smiles>

(7)<smiles>CC1=CCC2C1[C@@H]1CCC(C)(C)C2CC1C(C)C</smiles>

(11)<smiles>CC(=O)OCC=C(C)CCC=C(C)C</smiles>

(4)<smiles>C=C[C@](C)(O)CCC=C(C)C</smiles>

(8)<smiles>CC12CCC(C)(CC1)C2(C)C</smiles>

(12) 
The bark oil was extracted from nine species of Goniothalamus which are G. clemensii, G. macrophyllus, $G$. malayanus, G. ridleyi, G. tapis, G. tapisoides, G. uvarioides, G. velutinus, and G. woodii. Seven species were found for its richness in oxygenated sesquiterpenes. They were $\alpha$-cadinol $(41.6 \%), \beta$-eudesmol $(32.2 \%), \gamma$ eudesmol (21.8\%), agarospirol (19.0\%), elemol (16.1\%), hedycaryol (13.6\%), $\alpha$-eudesmol (9.7\%), t-muurolol (9.1\%), (E)-nerolidol (9.1\%), cubenol (7.7\%), and (Z)-nerolidol (5.2\%). In addition, sesquiterpene hydrocarbons were also found in the bark oils of Malaysian Goniothalamus. They were revealed by the presence of $\beta$-selinene (6.1\%) found in the bark oil of G. velutinus. Furthermore, oxygenated monoterpenes were found in four local Goniothalamus bark oils. They were found in the bark oil of G. macrophyllus, $G$. ridleyi, G. tapis, and G. tapisoides. They were characterised by 1,8-cineole (47.9\%), terpinen-4-ol (42.7\%), linalool (15.8\%), $\alpha$-terpineol (10.0\%), and geraniol (9.7\%). Moreover, monoterpene hydrocarbons were also found as the major group components in four bark oils of Goniothalamus collected from Malaysia. (Z)- $\beta$ Ocimene (25.4\%) and limonene (7.5\%-12.7\%) were found in the bark oil of G. macrophyllus, G. ridleyi and G. tapis. Meanwhile, p-menthene (6.9\%), $\alpha$-pinene (6.6\%), and $p$-terpinene (6.6\%) were found in the bark oil of G. tapisoides. Geranyl acetate (11.1\%), an ester was the major component of G. macrophyllus bark oil. Aldehyde which is citronellal (6.3\%-12.9\%) and phenyl propanoid which is safrole (5.3\%-11.2\%) were found in the bark oil of $G$. ridleyi and G. tapis, respectively.

Five essential oils of Malaysian Goniothalamus have been extracted by using leaf parts which are $G$. andersonii, G. malayanus, G. tapis, G. tapisoides, and G. uvarioides. The leaf oils were dominated by sesquiterpene hydrocarbons, oxygenated sesquiterpenes, monoterpene hydrocarbons, and oxygenated monoterpenes. Four out of five species have sesquiterpene hydrocarbons as their major components. They were identified as $\beta$-selinene (33.6\%), $\alpha$-copaene (23.8\%), $\beta$-cubebene (15.2\%), $\beta$-caryophyllene (14.4\%), and $\alpha$-muurolene (4.8\%). In addition, oxygenated sesquiterpenes were also found in the leaf oil which was guaiol (28.6\%), elemol (19.6\%), viridiflorol (13.1\%), and epi-globulol (7.7\%). Moreover, oxygenated monoterpenes which were 1,8-cineole (7.6\%-79.0\%), linalool (18.5\%), and $\alpha$-terpineol (4.4\%) were found in two leaf oils identified as $G$. tapis and $G$ tapisoides. Besides, $\alpha$-pinene $(9.6 \%)$ was the only monoterpene hydrocarbon found from the leaf oil of $G$. tapisoides.

The root oils of Malaysian Goniothalamus have been extracted from five species which were identified as $G$. macrophyllus, G. malayanus, G. tapis, G. tapisoides, and G. uvarioides. The components can be classified as oxygenated sesquiterpenes, sesquiterpene hydrocarbons, oxygenated monoterpenes, monoterpene hydrocarbons and ester. The root oil of $G$. malayanus was dominated by oxygenated sesquiterpenes which were characterized by elemol (5.1\%), (E)-nerolidol (6.5\%), and $\gamma$-eudesmol (18.8\%). $\beta$-eudesmol (5.0\%-27.8\%) and $\alpha$-eudesmol (5.7\%-6.0\%) was found in both $G$. malayanus and $G$. tapis root oils. Next, sesquiterpene hydrocarbons found in the root oils were cyperene (9.8\%-16.2\%), $\alpha$-copaene (8.7\%), $\beta$-elemene (5.3\%), and $\gamma$-amorphene (5.2\%). Furthermore, oxygenated monoterpenes were found in the root oils of $G$. tapisoides and $G$. uvarioides which are 1,8-cineole (14.0\%-56.1\%), terpinen-4-ol (19.6\%-39.5\%), and $\alpha$-terpineol (6.3\%). Monoterpene hydrocarbons which were camphene $(7.5 \%), \gamma$-terpinene $(5.7 \%)$, and p-cymene $(5.1 \%)$ were found in the root oils of G. macrophyllus, G. tapisoides, and G. uvarioides. Last but not least, geranyl acetate (9.4\%) which is an ester was found in the root oil of G. macrophyllus. Meanwhile, the fruit oil only reported from $G$. ridleyii with $\beta$-cubebene, elemol, and $\beta$-eudesmol as the major components (Jusoh et al., 2015).

\section{Biological activities}

Although many members of the genus Goniothalamus are renowned for their valuable essential oils, the genus is still poorly explored as far as its biological activities of essential oil are concerned. A search of the literature revealed the occurrence of antimicrobial and antiplatelet aggregation and platelet-activating factor 
(PAF) receptor antagonistic activities that have been reported from the Malaysian Goniothalamus essential oils.

The antimicrobial activity of root and twig oils of G. macrophyllus has been reported by Ghani et al. (2010). The oils were found to demonstrate the notable antimicrobial activity with the MIC values below the cut-off point of $1 \mathrm{mg} / \mathrm{mL}$. The root oils were considerably more active than the twig oil in inhibiting all the bacterial and fungal strains except $P$. aeruginosa. The root oils had the strongest inhibitory effect against VISA24, $S$. epidermidis and C. albicans with the MIC values of $0.3 \mathrm{mg} / \mathrm{mL}$. The twig oils demonstrated moderate to weak activity toward all bacterial strains tested with the MIC values ranging from 2.5 to $5 \mathrm{mg} / \mathrm{mL}$. Both dermatophytes, $T$. rubrum and $M$. cannis however showed similar susceptibility to the twig and root oils with the MIC values of $2.5 \mathrm{mg} / \mathrm{mL}$.

Moharam et al. (2010) reported antiplatelet aggregation and platelet-activating factor (PAF) receptor antagonistic activity against nine Goniothalamus essential oils (G. velutinus, G. woodii, G. clemensii, G. tapis and $G$. tapisoides). The bark oil of $G$. velutinus was the most effective sample as it inhibited both arachidonic acid $(A A)$ and $A D P$-induced platelet aggregation with $I C_{50}$ values of 93.6 and $87.7 \mu \mathrm{g} / \mathrm{mL}$, respectively. Among the studied oils, the bark oils of $G$. clemensii, G. woodii, G. velutinus and the root oil of $G$. tapis showed significant inhibitory effects on PAF receptor binding, with $\mathrm{IC}_{50}$ values ranging from 3.5 to $10.5 \mu \mathrm{g} / \mathrm{mL}$.

\section{Multivariate statistical analysis}

Ten Malaysian Goniothalamus species (28 samples) have been selected for this study which are G. andersonii, G. clemensii, G. macrophyllus, G. malayanus, G. ridleyi, G. tapis, G. tapisoides, G. uvarioides, G. velutinus, and G. woodii. Information on plant materials used for multivariate statistical analysis as shown in Table 3.

Table 3. Information on plant materials used for multivariate statistical analysis.

\begin{tabular}{|c|c|c|c|c|}
\hline Species & Collection Site & Parts & Lable & References \\
\hline G. clemensii & Sematan, Sarawak & Bark & GCB & Moharam et al., 2010 \\
\hline G. macrophyllus 1 & Fraser Hill, Pahang & Bark & GMB1 & Jantan et al., 2005 \\
\hline G. macrophyllus 2 & Lawas, Sarawak & Bark & GMB2 & Jantan et al., 2005 \\
\hline G. malayanus & Kota Samarahan, Sarawak & Bark & GLB & Jantan et al., 2005 \\
\hline G. ridleyii 1 (Fresh) & Gua Musang, Kelantan & Bark & GRB1 & Jusoh et al., 2015 \\
\hline G. ridleyii 2 (Dry) & Gua Musang, Kelantan & Bark & GRB2 & Jusoh et al., 2015 \\
\hline G. tapis 1 & Lawas, Sarawak & Bark & GTB1 & Moharam et al., 2010 \\
\hline G. tapis 2 & Lawas, Sarawak & Bark & GTB2 & Ahmad et al., 2010 \\
\hline G. tapisoides 1 & Sematan, Sarawak & Bark & GOB1 & Moharam et al., 2010 \\
\hline G. tapisoides 2 & Lawas, Sarawak & Bark & GOB2 & Ahmad et al., 2010 \\
\hline G. uvaroides & Merapok, Sarawak & Bark & GUB & Jantan et al., 2005 \\
\hline G. velutinus & Sematan, Sarawak & Bark & GVB & Moharam et al., 2010 \\
\hline G. woodii & Sematan, Sarawak & Bark & GWB & Moharam et al., 2010 \\
\hline G. andersonii & Kota Samarahan, Sarawak & Leaf & GAL & Jantan et al., 2005 \\
\hline G. malayanus 1 & Kota Samarahan, Sarawak & Leaf & GLL1 & Jantan et al., 2005 \\
\hline G. malayanus 2 & Kota Samarahan, Sarawak & Leaf & GLL2 & Jantan et al., 2005 \\
\hline G. tapis 1 & Lawas, Sarawak & Leaf & GTL1 & Moharam et al., 2010 \\
\hline G. tapis 2 & Lawas, Sarawak & Leaf & GTL2 & Ahmad et al., 2010 \\
\hline G. tapisoides 1 & Sematan, Sarawak & Leaf & GOL1 & Moharam et al., 2010 \\
\hline G. tapisoides 2 & Sematan, Sarawak & Leaf & GOL2 & Ahmad et al., 2010 \\
\hline G. uvarioides & Merapok, Sarawak & Leaf & GUL & Jantan et al., 2005 \\
\hline
\end{tabular}




\begin{tabular}{|c|c|c|c|c|}
\hline G. macrophyllus & Pasoh Negeri Sembilan & Root & GMR & Ghani et al., 2010 \\
\hline G. malayanus 1 & Kota Samarahan, Sarawak & Root & GLR1 & Jantan et al., 2005 \\
\hline G. malayanus 2 & Kota Samarahan, Sarawak & Root & GLR2 & Jantan et al., 2005 \\
\hline G. tapis & Lawas, Sarawak & Root & GTR & Ahmad et al., 2010 \\
\hline G. tapisoides 1 & Sematan, Sarawak & Root & GOR1 & Moharam et al., 2010 \\
\hline G. tapisoides 2 & Sematan, Sarawak & Root & GOR2 & Ahmad et al., 2010 \\
\hline G. uvaroides & Merapok, Sarawak & Root & GUR & Jantan et al., 2005 \\
\hline
\end{tabular}

Principal component analysis (PCA) and hierarchical cluster analysis (HCA) were used to characterize their essential oils components. The components common to all essential oils were used to determine the similarity among species with a CA performed with the software Statistica 7.0. The Unweighted Pair Group Method with Arithmetic Mean (UPGMA) was used to cluster groups based on Euclidean distance. The PCA was carried out with the software Statistica 7.0. PCA was used to reveal interrelationships among the ten species of the genus Goniothalamus based on the essential oil common components of these species (Wickramagamage, 2010; Shaharudin et al., 2013, 2018).

The HCA analysis revealed four distinct groups for each leaf bark, leaf, and root oils, based on the Euclidian distance as illustrated in Figure 2, Figure 3 and Figure 4, respectively. For Goniothalamus bark oils, the first group, Cluster I consisted of G. clemensii, G. velutinus and G. woodii. Cluster II included G. macrophyllus (1 and 2 ) and $G$. tapisoides (1 and 2). Meanwhile, Cluster III consisted of G. malayanus and G. uvaroides, whereas Cluster IV contained G. ridleyi (1 and 2) and G. tapis (1 and 2). For Goniothalamus leaf oil, the first group, Cluster I consisted of $G$. andersonii and G. uvarioides. Cluster II included G. malayanus (1 and 2), whereas Cluster III consisted of G. tapis (1 and 2). In addition, Cluster IV consisted of G. tapisoides (1 and 2). For Goniothalamus root oils, the first group, Cluster I consisted of $G$ macrophyllus, while Cluster II comprised $G$. tapisoides (1 and 2) and G. uvaroides. Meanwhile, Cluster III consisted of G. malayanus (1 and 2), whereas Cluster IV contained G. tapis.

Furthermore, to evaluate the accuracy of this classification, the cluster obtained was confirmed by PCA analysis. Similarly, the bark, leaf and roots oils of Goniothalamus were divided into four groups each, Cluster I-IV. The results were obtained by PCA based on forty-one (bark oil), thirty-four (leaf oil), and thirty-one (root oil) chemical components. Three factors explained 51.50\% (bark oil), 59.76\% (leaf oil), and 65.99\% (root oil) of accumulated variation of the data analysed. The first three are considered the most important as they represent $\geq 50 \%$ of the accumulated variation. Furthermore, these results also may be correlated with other factors involving a genetic determination that could also be modulated by biotic pressures, volatile constituents during flowering influenced by pollinators, and during the vegetative phase by pathogens and herbivores, or differences in environmental conditions (Silva et al., 2007). Thus, the variation pattern in essential oil composition may reflect selective pressures in different ecological and geographical environments (ecotypes). 
Figure 2. PCA and UPGMA analyses of the composition of Malaysian Goniothalamus bark oils

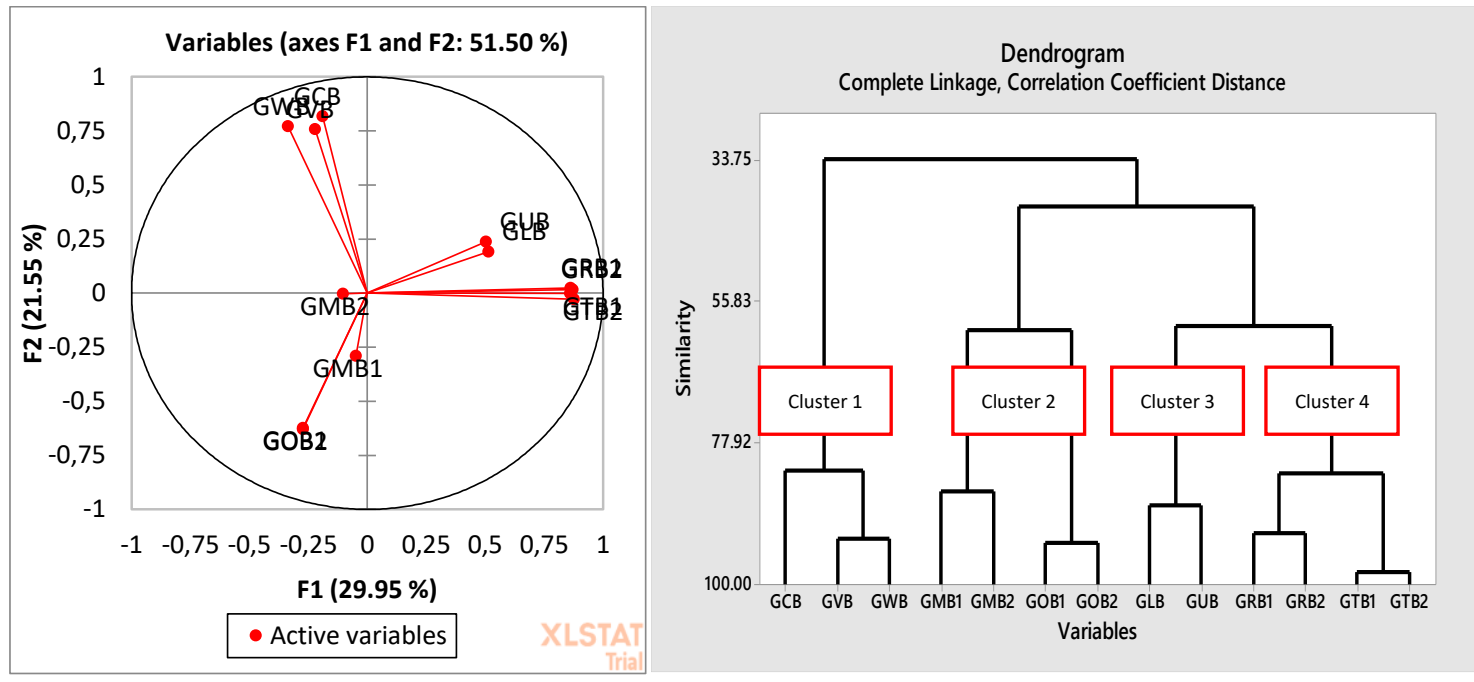

Figure 3. PCA and UPGMA analyses of the composition of Malaysian Goniothalamus leaf oils
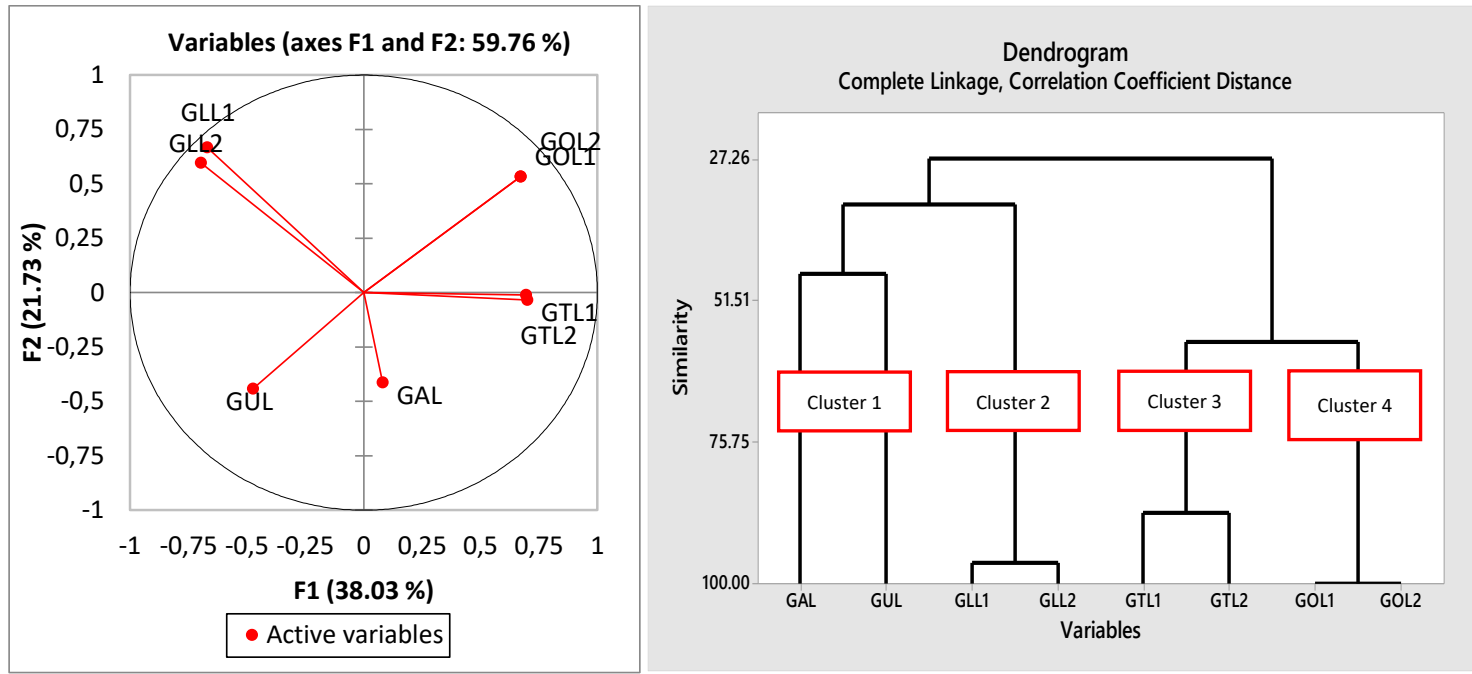

Figure 4. PCA and UPGMA analyses of the composition of Malaysian Goniothalamus root oils
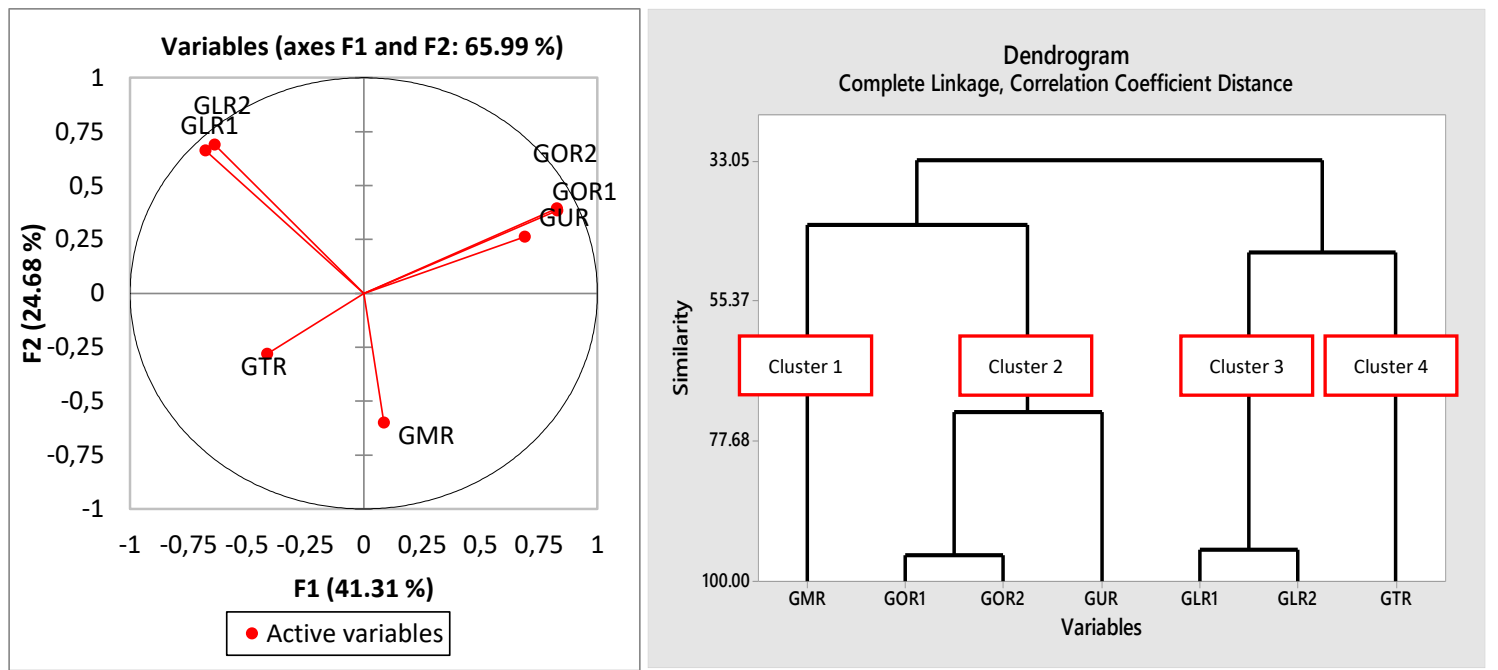


\section{Conclusion}

In conclusion, our study reports a review of Malaysian Goniothalamus essential oils and their chemical variability. This information is critical when selecting species with economic potential for the pharmaceutical and cosmetics industry. In addition, the multivariate data analysis may be used as quality control tools for the identification and characterization of essential oils from different Goniothalamus species that are to be utilized as raw materials in traditional herbal products. Further studies need to be carried out to determine fingerprints and chemical compositions of other Goniothalamus species and those collected from different origins.

\section{ACKNOWLEDGMENT}

The authors would like to thank the Department of Chemistry, Faculty of Science and Mathematics, Universiti Pendidikan Sultan Idris for research facilities.

\section{CONFLICTS OF INTEREST}

The authors have no conflicts of interest to declare.

\section{REFERENCES}

Ahmad, B. F., Moharm, B. A., Jantan, I. (2010). A comparative study of the constituents of the essential oils of Goniothalamus tapis Miq. and G. tapisoides Mat Salleh from Borneo. Journal of Essential Oil Research, $22(6), 499-502$.

Ahmad, F. B., Tukol, W. A., Omar, S., Sharif, A. M. (1991). 5-Acetyl goniothalamin, a styryl dihydropyrone from Goniothalamus uvaroides. Phytochemistry, 30(7), 2430-2431.

Akter, N., Habiba, N. A., Afrin, T., Ferdushi, M., Islam, S., Masud, K. N. A. (2018). Study of cytotoxic and thrombolytic activity of Goniothalamus sesquipedalis in different extracts. International Journal of Research in Pharmacy and Pharmaceutical Sciences, 3(6), 19-22.

Alkofahi, A., Rupprecht, J., Smith, D. L., Chang, C.J., Mclaughlin, J. L. (1998). Goniothalamin and annonacin: Bioactive acetogenins from Goniothamalus giganteus (Annonaceae). Experientia, 44, 83-85.

Anary, P. M., Brandao E., Déborah, Y., Santos, A. C. (2016). Chapter 9 - Nutritional value of the pulp of different sugar apple cultivars (Annona squamosa L.). Nutritional Composition of Fruit Cultivars.

Aslam, M. S., Ahmad, M. S., Mamat, A. S., Ahmad, M. Z., Salam, F. (2016). Goniothalamus: phytochemical and ethnobotanical review. Recent Advances in Biology and Medicine, 2, 34-47.

Burger, P., Plainfossé, H., Brochet, X., Chemat, F., Fernandez, X. (2019). Extraction of natural fragrance ingredients: history overview and future trends. Chemistry and Biodiversity, 16(10), e1900424.

Burkill, I. H. (1966). A Dictionary of Economic Products of the Malay Peninsula. Ministry of Agriculture and Co-operative: Kuala Lumpur, Malaysia.

Duc, L. V., Thanh, T. B., Thanh, H. N., Tien, V. N. (2016). Chemical constituents and cytotoxic effect from the barks of Goniothalamus chinensis Merr. \& Chun. growing in Vietnam. Journal of Applied Pharmaceutical Science, 6(4), 1-5.

Funnimid, N., Pompimon, W., Nuntasaen, N. (2019). In vitro evaluation of crude extracts and isolated compounds from Goniothalamus rongklanus and Goniothalamus latestigma for bioactive properties. Journal of Natural Remedies, 19(3), 146-152.

Ghani, S. H. A., Nor Azah, M. A., Mastura, M., Mailina, J., Saiful, J. A., Muhajir, H., Puad, A. M. (2010). Chemical constituents and antimicrobial activity of Goniothalamus macrophyllus (Annonaceae) from Pasoh Forest Reserve, Malaysia. African Journal of Biotechnology, 9(34), 5511-5515. 
Iqbal, E., Salim, K. A., Lim, L. B. L. (2015). Phytochemical screening, total phenolics and antioxidant activities of bark and leaf extracts of Goniothalamus velutinus (Airy Shaw) from Brunei Darussalam. Journal of King Saud University - Science, $27(3), 224-232$.

Inayat-Hussain, S. H., Osman, A. B., Din, L. B., Ali, A. M., Snowden, R. T. (1999). Caspases-3 and-7 are activated in Goniothalamin-induced apoptosis in human Jurkat T-cells. Federation of European Biochemical Societies Letters, 456(3), 379-383.

Jantan, I., Ahmad, A. S., Ahmad, F. (2005). A comparative study of the essential oils of four Goniothalamus species. Acta Horticulturae, 677, 27-36.

Jiang, M. M., Feng, Y. F., Gao, H., Zhang, X., Tang, J. S., Yao, X. S. (2011). Three new bis styryllactones from Goniothalamus cheliensis. Fitoterapia, 82(4), 524-527.

Jusoh, S., Ahmad, F., Din, L. B., Zakaria, Z. (2015). Essential oils from different parts of Goniothalamus ridleyi plant. Sains Malaysiana, 44(11), 1579-1585.

Kim, R. P. T., Bihud, V., Mohamad, K., Leong, K. H., Mohamad, J., Ahmad, F., Hazni, H., Kasim, N., Halim, S. N. A., Awang, K. (2013). Cytotoxic and antioxidant compounds from the stem bark of Goniothalamus tapisoides mat salleh. Molecules, $18(1), 128-139$.

Mahiwan, C., Buayairaksa, M., Nuntasaen, N., Meepowpan, P., Pompimo, W. (2013). Potential cancer chemopreventive activity of styryllactones from Goniothalamus marcanii. American Journal of Applied Sciences, 10, 112-116.

Moharam, B. A., Jantan, I., Ahmad, F. B., Jalil, J. (2010). Antiplatelet aggregation and platelet activating factor (PAF) receptor antagonistic activities of the essential oils of five Goniothalamus species. Molecules, 15(8), 5124-5138.

Moharam, B. A., Jantan, I., Jalil, J., Ahmad, F. B. (2012). Inhibitory effect of compounds from Goniothalamus tapis Miq. and Goniothalamus uvaroides king on platelet activating factor receptor binding. Phytotherapy Research, 26(5), 687691.

Nielsen, I. (1993). Flowers of Annonaceae: morphology, classification and evolution. Nordic Journal of Botany, $13(5), 1$.

Quisumbing, E. (1951). Medicinal Plants of the Philippines. Bureau of Printing, Manila.

Salleh, W. M. N. H. W., Ahmad, F., Khong, H. Y. (2014a). Chemical composition of Piper stylosum Miq. and Piper ribesioides Wall. essential oils and their antioxidant, antimicrobial and tyrosinase inhibition activities. Boletin Latinoamericano y del Caribe de Plantas, 13(5), 488-497.

Salleh, W. M. N. H. W., Ahmad, F., Khong, H. Y. (2014b). Chemical compositions and antimicrobial activity of the essential oils of Piper abbreviatum, P. erecticaule and P. lanatum (Piperaceae). Natural Product Communications, 9(12), 17951798.

Salleh, W. M. N. H. W., Ahmad, F., Khong, H. Y., Zulkifli, R. M. (2015a). Chemical compositions and biological activities of essential oils of Beilschmiedia glabra. Natural Product Communications, 10(7), 1297-1300.

Salleh, W. M. N. H. W., Ahmad, F., Khong, H. Y. (2015b). Antioxidant and anticholinesterase activities of essential oils of Cinnamomum griffithii and C. macrocarpum. Natural Product Communications, 10(8), 1465-1468.

Salleh, W. M. N. H. W., Kamil, F., Ahmad, F., Sirat, H. M. (2015c). Antioxidant and anti-inflammatory activities of essential oil and extracts of Piper miniatum. Natural Product Communications, 10(11), 2005-2008.

Salleh, W. M. N. H. W., Ahmad, F., Khong, H. Y., Zulkifli, R. M. (2016a). Essential oil composition of Malaysian Lauraceae: A mini review. Journal of Pharmaceutical Sicences, 22(1), 60-67.

Salleh, W. M. N. H. W., Ahmad, F., Khong, H. Y., Zulkifli, R. M. (2016b). Comparative study of the essential oils of three Beilschmiedia species and their biological activities. International Journal of Food Science and Technology, 51, $240-249$. 
Salleh, W. M. N. H. W., Ahmad, F., Khong, H. Y., Zulkifli, R. M. (2016c). Chemical composition and biological activities of essential oil of Beilschmiedia pulverulenta. Pharmaceutical Biology, 54(2), 322-330.

Shaharudin, S. M., Ahmad, N., Yusof, F. (2013). Improved cluster partition in principal component analysis guided clustering. International Journal of Computer Applications, 75, 22-25.

Shaharudin, S. M., Ahmad, N., Zainuddin, N. H., Mohamed, N. S. (2018). Identification of rainfall patterns on hydrological simulation using Robust Principal Component Analysis. Indonesian Journal of Electrical Engineering and Computer Science, 11, 1162-1167.

Silva, F. G., Oliveira, C. B. A., Pinto, J. E. B. P., Nascimento, V. E., Santos, S. C., Seraphin, J. C., Ferri, P. H. (2007). Seasonal variability in the essential oils of wild and cultivated Baccharis trimera. Journal of the Brazilian Chemical Society, 18, 990997.

Wiart, C. (2006). Medicinal Plants of Asia and the Pacific. Taylor \& Francis, Boca Raton, London, New York.

Wiart, C. (2007) Goniothalamus species: a source of drugs for the treatment of cancers and bacterial infections? Evidence-Based Complementary and Alternative Medicine, 4, 299-311.

Wickramagamage, P. (2010). Seasonality and spatial pattern of rainfall of Sri Lanka: Exploratory factor analysis. International Journal of Climatology, 30, 1235-1245.

Winska, K., Mączka, W., Lyczko, J., Grabarczyk, M., Czubaszek, A., Szumny, A. (2019). essential oils as antimicrobial agents-myth or real alternative? Molecules, 24(11), 2130.

Wu, Y. C., Duh, C. Y., Chang, F. R., Chang, G. Y., Wang, S. K. (1991). The crystal structure and cytotoxicity of goniodiol-7monoacetate from Goniothalamus amuyon. Journal of Natural Products, 54(4), 1077-1081. 